\title{
L-Band In-Line Remote Amplification for an Extended WDM/PON Ring Architecture
}

\author{
Sotiria Chatzi*, Ioannis Tomkos, José A. Lazáro*, Josep Prat*. \\ Athens Information Technology, 19,5 km Markopoulou Ave., 19002 Athens ,Greece \\ * Universitat Politècnica de Catalunya, Jordi Girona 1-3, 08034 Barcelona, Spain \\ Tel: (0030) 210668 2732,Fax: (0030) 210668 2729,e-mail: soch@ait.edu.gr
}

\begin{abstract}
In this paper, a study on a fully passive WDM/PON ring architecture which reaches $19 \mathrm{~km}$ distance, while serving more than 1000 users with symmetric several hundred Mbit/s per user is presented. The design is based on SARDANA (Scalable Advanced Ring -based Passive Dense Access Network Architecture) and was generated as an alternative solution, with its novelty laying on the use of L-band in line remote amplification. The simulation of an extended access WDM/TDM PON formed in a double fibre ring with single fiber trees was created. The network's operation has been tested for several conditions of usage (i.e. maximum number of users, transmission power and pump power) and an optimization of the design has been performed. The target was to achieve, with given transmission and pump power, the reach of more then 1000 users with input power high enough to permit the use of an RSOA at the ONU. The use of L-band signals and special doped fiber has permitted the effective use of RSOAs in the total of the end users.
\end{abstract}

Keywords: in-line amplification, L- band, remote node, pump power, symmetric design, frequency allocation.

\section{INTRODUCTION}

Next generation Long Reach High Capacity Passive Optical Networks can serve simultaneously many subscribers with bandwidth hungry applications and at the same time cover longer distances with the goal to reduce significantly the capital and operational costs. Therefore efforts have been made by research projects such as SARDANA [1], PIEMAN [2] and GigaWam to expand the limits of PON while providing high bit rate connectivity.

Our approach, focusing on amplification and more specifically on in-line amplification using for transmission the L-band, is based and tested in the Scalable Advanced Ring-based passive Dense Access Network Architecture (SARDANA). The goal is to achieve, with given transmission and pump power, the longest reach possible from the Optical Line Terminal (OLT) to the furthest user, while serving more than 1000 users and maintaining the signal in an adequate level. In the scenario examined the signal level as well as the OSNR obtained, allow the use at the customer's end terminal of a Reflective Semiconductor Optical Amplifier (RSOA) which comprises of a cost effective device.

SARDANA consists of an innovative ring-based design that attempts to serve more than 1000 users with symmetric bandwidth of several hundred of Mbit/s per user, in distances reaching a maximum of $100 \mathrm{~km}$ (in case of resiliency), and shared feeder rates up to 10Gbit/s. It comprises a scalable and extended access WDM/TDM PON, formed in a double fibre ring and secondary single fiber trees [3]. One ring is dedicated to the transmission of downstream signals and the other to the transmission of the upstream ones. Each fibre ring is designed to operate alike in both directions in order to provide resiliency in case of a fiber cut.

The Central Office (CO) contains the Optical Line Terminal (OLT), thus the transmitters and receivers required to serve the network. The laser providing the pump signal is collocated with the OLT [3]. The CO is used to house all the active components of the network and therefore SARDANA can be characterized as a fully passive architecture.

The scalability of the network is achieved with the use of passive Remote Nodes (RN) and remote amplification. The RNs are used mainly to perform the add/drop function of dedicated wavelengths to each tree, with the use of filters and splitters, whose combination selects two wavelengths and distributes them to two independent single fiber trees per RN [3]. The trees' splitting ratio can vary according to the needs of the network in means of number of users. Proposed splitting ratios for an urban deployment are 1:32, 1:64, 1:128, so the variation of served users is flexible, but its increase is achieved in expense of additional losses.

The signal is detected in the optical network unit (ONU) by an RSOA. The RSOA has a double operation in the network. Besides detecting the downstream data, it modulates the optical carrier sent along with the downstream data, with the user's upstream data [4].

The scalability of the network permits the implementation of several deployments, whose planning depends on the population density and the area considered. These characteristics determine the final number of the RNs as well as the distances among them. However, in every approach the need of remote amplification is necessary in order to have the appropriate input level in the ONU and to maximize the users while counterbalancing for the power losses throughout the network. SARDANA covers this necessity by means of amplification in the drop part of the RN. 


\section{PARAMETERS}

The parameters taken under consideration are: the number of RNs, the signal power, the pump power, the total length of the fiber between the OLT and the final user -which could be considered as the sum of the fiber distances from the OLT to the RN and the tree fiber length-, the losses induced by the RN, the splitting ratio of the tree, the length of the remotely amplified EDF, the percentage of the total pump power used in each RN to pump the corresponding EDF, the sensitivity of the receivers and the wavelength allocation in the sense of appointing which frequencies will be dropped in each $\mathrm{RN}$ regarding their distance from the OLT.

\subsection{Number of RNs}

The number of RNs depends on the number of end users we want to cover. In an urban area a number of 16RNs per fiber ring is considered. Increase of the number of nodes corresponds to increase of the users served and decrease of the power budget.

\subsection{Signal power value}

The downstream signal power's value has been set in $10 \mathrm{dBm}$. The reason for that is that with the power link budget performed, the design of in-line amplification requires $10 \mathrm{dBm}$ initial power in order to reach the users of the first tree, since no amplification is performed for this frequency. Moreover the use of a stronger signal power was avoided, so as to prevent emergence of non linear effects at furthest propagation, as well as for restraining OPEX. The upstream signals' value is set to $0 \mathrm{dBm}$ in order to comply with the capabilities of an RSOA.

\subsection{Pump power value $\&$ distribution}

The operation of in-line amplification design was tested for two values of pump power, 31 and $39 \mathrm{dBm}$ respectively. Increase of the pump power makes the network more flexible in terms of users served. The reason is that it allows a more effective amplification thus greater gain, thereby an increase in the power budget is achieved. Nevertheless we examined the use of $31 \mathrm{dBm}$, because a smaller pump power complies better with safety regulations as far as the CO is concerned. It should be marked however, in regard of safety regulations, that since the pump circulates only in the upstream fiber ring, there is no danger that it could come to the reach of the end user. The pump is counter propagating with the upstream signals as aforementioned, but in this investigation the loss due to pump depletion is considered negligible, because of the low signal power.

Pump power is distributed in each RN to be used in amplification of both the downstream and the upstream propagating signals. The distribution is implemented by using different percentages in each RN for the amplification of the downstream signals, while the portion used for the amplification of the upstream signals is invariable. As far as the downstream propagation is concerned, the required power was determined by the needs of each RN for amplification. In some distances the signal had the power required to reach the customers' ONU without amplification, some RNs were excluded. A conservative approach was considered on the use of the pump so that it would be adequately distributed to all amplification stages.

\subsection{Losses induced by the RN}

Each RN includes filters that perform Add \& Drop operation. In the cases of RNs that include EDFs for in-line amplification, the use of splitters and WDM couplers is additionally necessary. The filters used along with the splitters and the WDM couplers introduce losses at the RN. Losses are also induced by the WDM couplers and the attenuation of the fiber along the path to the end user. Their sum is $4.6 \mathrm{~dB}$. All these losses have to be counterbalanced by the gain of the EDF.

\subsection{Splitting ratio}

As aforementioned the splitting ratio of the tree can vary from 1:32, 1:64 and 1:128 for an urban deployment. Since each node serves two trees, each with a different $\lambda$, these splitting ratios are interpreted into 64,128 and 256 users per RN respectively. It is obvious that increasing the splitting ratio, the number of end users is increasing accordingly, one should take into account though, that this happens in expense of power losses induced to the network. In the scenario presented a splitting ratio of 1:32 has been used.

\subsection{EDF length}

The length of the remotely pumped EDF affects the amount of gain achieved, the portion of the pump power consumed, as well as the OSNR. Therefore a careful choice has been made on the lengths of the EDFs used in each $\mathrm{RN}$ in respect of its position in the network (distance from the OLT) in order to counterbalance for all the aforementioned variables. Different fiber lengths have been used in the downstream ring, symmetrically placed though (i.e. same EDF lengths used in the first and last RN, so as to preserve a symmetrical design) in order to adapt in the case of resiliency operation (for example a fiber cut between the OLT and the first or the last RN). Furthermore due to the elaborate design some RNs could be excluded since the signal had the necessary power to serve the corresponding trees and no EDFs were placed in their interior. In the upstream all RNs were 
equipped with an EDF of the same length, but carefully optimized in terms of low pump power consumption and OSNR.

\subsection{Receiver's sensitivity}

We consider the use of an RSOA. A power value of $-20 \mathrm{dBm}$ is considered necessary in order to both obtain the downstream data and at the same time use the optical carrier for modulation with the upstream signal and achieve an output of $0 \mathrm{dBm}$. Transmission with low input powers have been already demonstrated [5]. Finally the receiver at the OLT is considered to have a sensitivity of $-28 \mathrm{dBm}$.

\subsection{Frequency allocation}

32 channels have been considered, with $100 \mathrm{GHz}$ spacing that range within the L-band from $187.4 \mathrm{THz}$ to $190.5 \mathrm{THz}$. In order to achieve the same gain through the amplification stages for all wavelengths, an optimum design per RN and per in-line amplifier is required. The main strategy in this optimum design has been to consider the worst case of resiliency and reserve those frequencies that can have higher gain for reaching the distant nodes. The gain of the EDF is not flat for all wavelengths, but exhibits a gain tilt which favors some of them in terms of provided gain. As the pump power fades out over longer distances, these channels that have received higher gain in the previous stages will be able to reach longer distances. Since this effect is evident in low frequencies, these must be allocated to the furthest $\mathrm{RNs}$ in the resiliency case, which are respectively $\mathrm{RN}_{16}$ and $\mathrm{RN}_{1}$, depending on the connection that falls down, either the connection from $\mathrm{RN}_{16}$ to OLT or from $\mathrm{RN}_{1}$ to OLT. Frequency allocation is shown in the Table 1.

Table 1. Frequency allocation.

\begin{tabular}{|c|c|c|c|c|c|c|c|c|c|c|c|c|c|c|c|c|}
\hline $\mathbf{R N}$ & 1 & 2 & 3 & 4 & 5 & 6 & 7 & 8 & 9 & 10 & 11 & 12 & 13 & 14 & 15 & 16 \\
\hline $\mathbf{f}_{\mathbf{1}}$ & 187.6 & 188 & 188.4 & 188.8 & 189.2 & 189.6 & 190 & 190.5 & 190.3 & 189.9 & 189.5 & 189.1 & 188.7 & 188.3 & 187.9 & 187.5 \\
\hline $\mathbf{f}_{\mathbf{2}}$ & 187.7 & 188.1 & 188.5 & 188.9 & 189.3 & 189.7 & 190.1 & 190.4 & 190.2 & 189.8 & 189.4 & 189 & 188.6 & 188.2 & 187.8 & 187.4 \\
\hline
\end{tabular}

\section{SET UP}

The set up is considered to be applied in an urban area, therefore it consists of $16 \mathrm{RNs}$, with a distance of $1 \mathrm{~km}$ among them, and an OLT connected with two fiber rings. One of them is used for downstream transmission, while in the other the upstream signals are counter propagating with the pump signal. Pump power is set to $31 \mathrm{dBm}$. The tree fiber has a $3 \mathrm{~km}$ length, so the maximum distance from the OLT to the end user is $19 \mathrm{~km}$. Considering the total passive losses of the network it is evident that these depend mainly on the splitting ratio at the distribution trees. In order to comply with the given values for transmitter-receiver power levels, the splitting ratio was set in 1:32 for each tree, which results in 1024 customers served.

The in-line amplification is realized in the RN. With the use of fixed filters and splitters two wavelengths are dropped in each RN. The EDF is placed among the two drop filters and before a 50/50 splitter used for resiliency. Each wavelength is then driven to a tree. The placement of the EDF among the drop filters creates the need to transmit in the downstream direction a signal with adequate power to reach the first tree (served by $\mathrm{RN}_{1}$ in normal operation or $\mathrm{RN}_{16}$ when operating in resiliency mode in the case of a fiber cut between the OLT and $\mathrm{RN}_{1}$ ) as the drop signal of the first tree in this design will not be amplified. An overview of the network and the $\mathrm{RN}$ design is presented in figure [1].

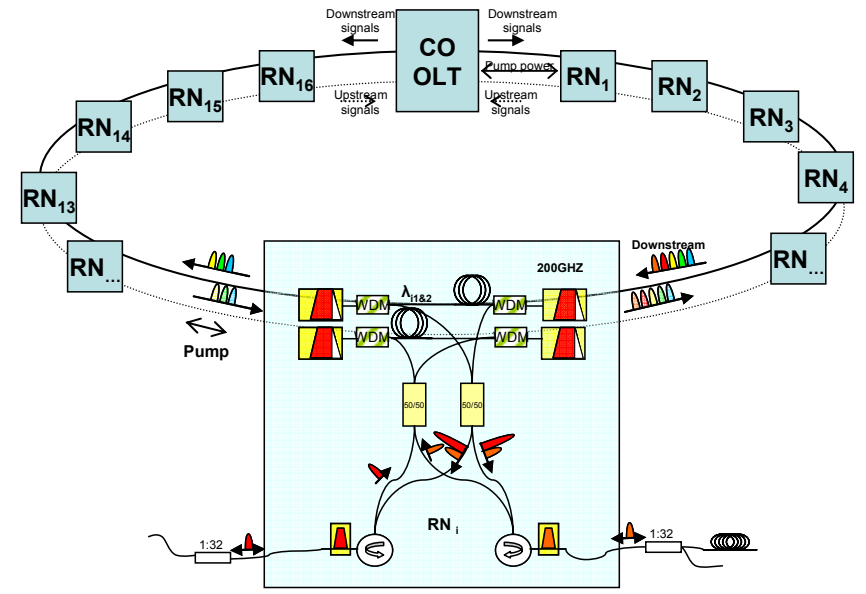

Figure 1. Network architecture and Remote Node comprising in line remote amplification. 


\section{RESULTS}

The study performed shows that the described architecture along with the use of L-band signals can serve 1024 end users with several hundreds of symmetric data rate in a maximum distance of $19 \mathrm{~km}$. More specifically for downstream as can be seen in figure [2], all the ONUs are reached with a signal power higher than $-20 \mathrm{dBm}$ which means, they could be based on RSOAs .

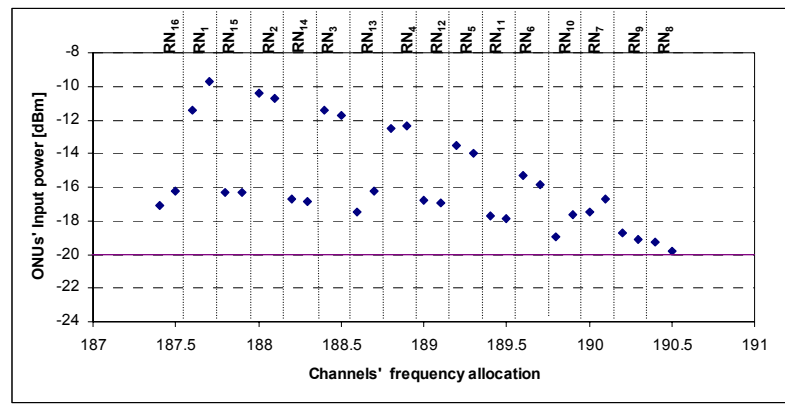

Figure 2.Downstream signals.

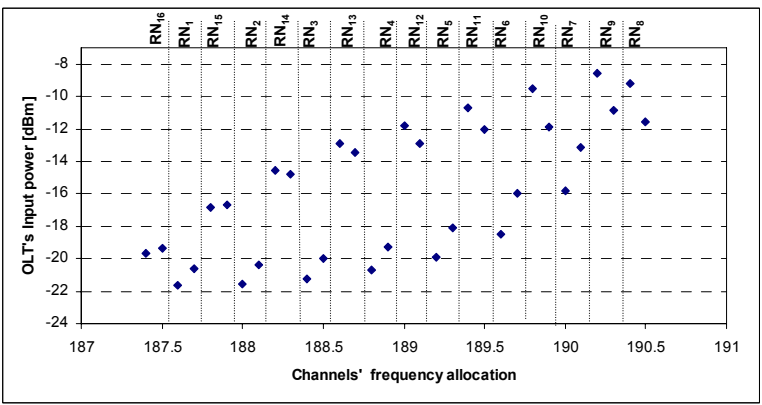

Figure 3. Upstream signals.

The upstream operation of the network is also ensured by the fact that the signal power level reaching the OLT is above $-28 \mathrm{dBm}$, as can be seen in figure [3], which is the OLT receiver's sensitivity. The gain of the special doped EDF has been experimentally investigated for different pumping power values and the results are depicted in figure [4].

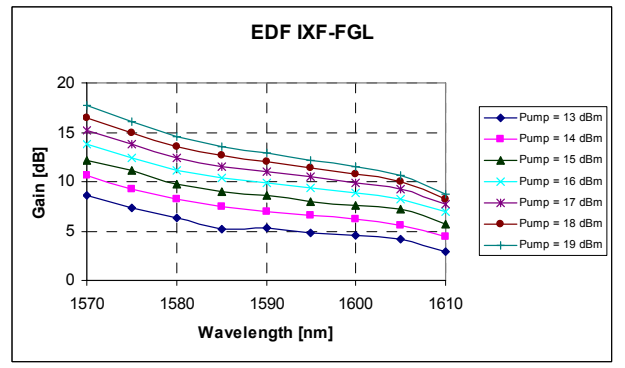

Figure 4. Characterization of the L-band amplifier in the RN.

\section{CONCLUSION}

In this paper we have shown that a network architecture based on SARDANA using L-band in line amplification can reach $19 \mathrm{~km}$ while serving 1024 users with several hundreds of Mbps and while all costumers are serviced with RSOA's based ONUs. The proposed remote amplification solution consists of a fully passive architecture which can expand the limits of already deployed passive infrastructures, using remote amplification. The low pump power required along with the extended use of RSOAs suggested, make this design very efficient in terms of OPEX. Furthermore the overall cost of the RSOAs and the different components of the network consist of a profitable solution in terms of CAPEX.

\section{ACKNOWLEDGEMENTS}

This work was supported by the European FP7 SARDANA and EURO-FOS projects.

\section{REFERENCES}

[1] J. Prat, et al.: Next generation architectures for optical access, ECOC 2006, pp.1-3, Sept. 2006.

[2] P.D. Townsend et al.: Long reach passive optical networks, in Proc. LEOS 2007, ThW1, pp.868-869, Oct. 2007.

[3] J.A. Lázaro et al.: Remotely Amplified SARDANA: Single-fibre-tree Advanced Ring-based Dense Access Network Architecture," in Proc. ECOC 2006, We3.P169, Cannes, 2006.

[4] J. Prat et al:: Optical network unit based on a bidirectional reflective semiconductor optical amplifier for fiber-to-the-home networks, IEEE Photonics Technology Letters, vol. 17, no1, pp. 250-252, Jan. 2005.

[5] Marco Presi et al:: A 80km reach fully passive WDM PON based on reflective ONUs, Optics Express, vol. 16, no. 23, pp. 19043-19048. 